\title{
"RED FLAGS" In Teenagers' Depression - A Family Doctors' Study and Point of View
}

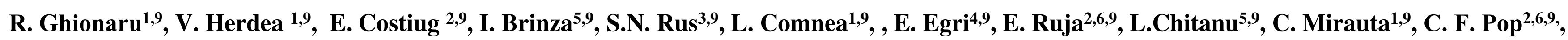
S. M. Cioc, ${ }^{1,9}$ C. Lanba ${ }^{1,9}$, P.Tarciuc ${ }^{1,9}$

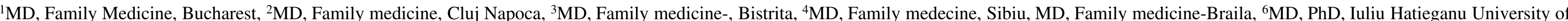

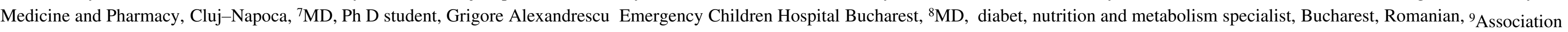
for Pediatric Education in Family Medicine (AREPMF), Corresponding author: Raluca Ghionaru raluca_ghionaru@yahoo.com

\section{Background}

Overall statistics show that depression may occur in up to $1 \%$ of preschoolers, $2-3 \%$ of school children and $5-8 \%$ of adolescents. In reality, however, depression is underdiagnosed, either by not recognizing early signs or by hiding them from the physician or the family. No data available for Romania.

\section{Aims}

Determining the susceptibility towards depression of adolescents from Romania by identifying risk factors "red flags"

\section{Method}

"Center for Epidemiological Studies Depression Scale for Children" (CES-DC), containing 20 self-assessment questions about depressive symptoms, was completed via an anonymous questionnaire:

\section{- 118 teenager respondents}

- female/male 2:1

- age between 15 and 18 years

We identified "red-flags" signs and symptoms of depression among high school students, but also the degree of addressability for therapy among this group age:

- CES-DC scores can be between 0 and 60 points.

- A score of 15 or above is interpreted as an indicator of the risk for depression.

- High score is associated with high risk.

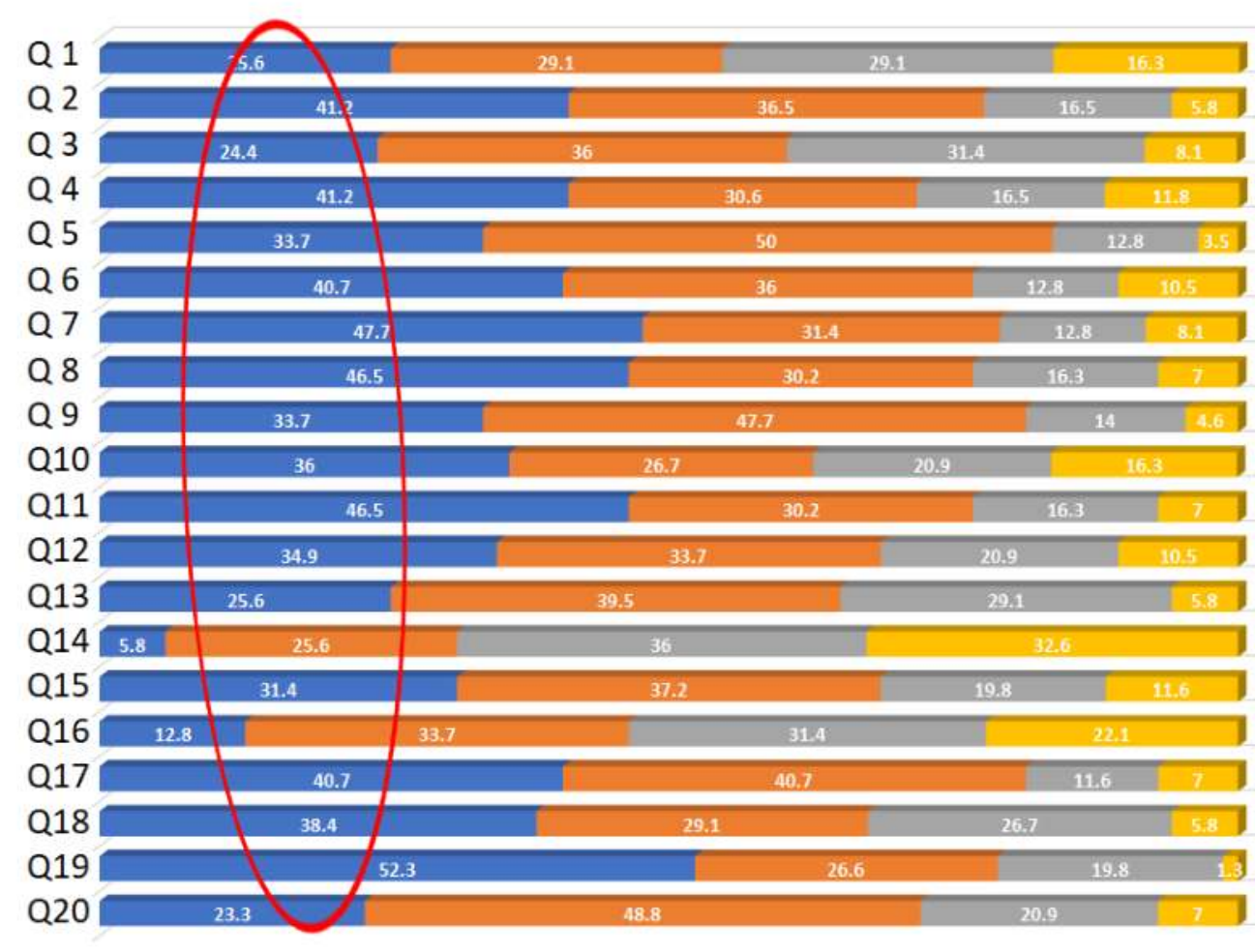

Fig. 1 The map of the answers to the questions for CES-DC form, in blue - considered normal responses, generating scoring below 15

\section{Results}

- Only $37.20 \%$ of respondents scored below 15 points.

- Girls are more prone to depression, with only $21.43 \%$ under 15 points , unlike boys (66.66\% under 15 points).

- Most scores over 15 were recorded at the age of 15 and 17 years.

- Teenagers avoid talking to a doctor when it comes to a mental health problem, preferring to hide reality from others.

\section{Discussions}

1. Study that evaluated the CES-DC found that scores do not necessarily match up to a DSM diagnosis, although it is a good psychometric tool for teenagers.

2. Screening for depression is a complex process and a score of 15 or higher on CES-DC must be followed by an additional professional assessment.

3. Social stigma, shame and pressure of the social group reduce addressability to physician or psychologist.

Addressability in case of emotional disorders

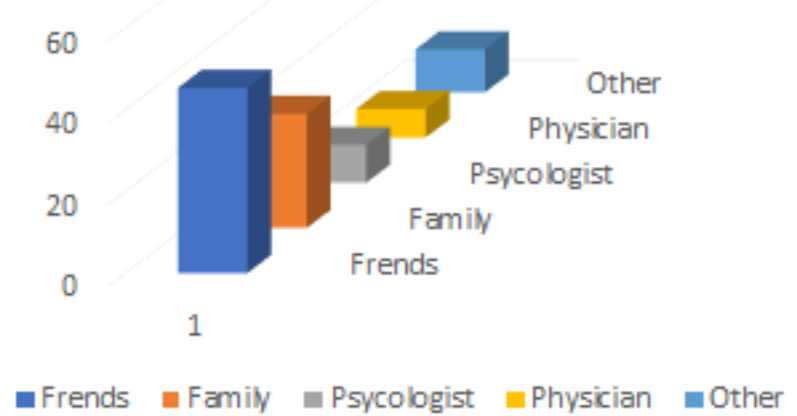

\begin{tabular}{|l|l|}
\hline Response & Percentage \\
\hline Frends & $45.3 \%$ \\
\hline Family & $27.9 \%$ \\
\hline Psycologist & $9.2 \%$ \\
\hline Physician & $7 \%$ \\
\hline Other & $10.5 \%$ \\
\hline
\end{tabular}

Fig. 2 Question: "In case of emotional disorders, refer to whom?"

\section{Conclusions}

1. Teenagers' depression is an important but underdiagnosed pathology and can endanger the personal development of the individual, this situation is favored by the lack of health education and stigma that cause adolescents to hide this pathology and do not address the doctor.

2. Health education programs in schools could help teenagers, parents and educators recognize the "red-flags" of depression.

3. There is a need for a family-educator-physician partnership so that the diagnosis and therapy could be early and efficient.

Keywords: depression, red-flegs, teenager 